\title{
Antitumor properties of dimethyl-celecoxib, a derivative of celecoxib that does not inhibit cyclooxygenase-2: implications for glioma therapy
}

\author{
AXel H. Schönthal, Ph.D. \\ Department of Molecular Microbiology and Immunology, Keck School of Medicine, \\ University of Southern California, Los Angeles, California
}

\begin{abstract}
$\checkmark$ Celecoxib (Celebrex) appears to be unique among the class of selective COX-2 inhibitors (coxibs), because this particular compound exerts a second function that is independent of its celebrated ability to inhibit COX-2. This second function is the potential to inhibit cell proliferation and stimulate apoptotic cell death at much lower concentrations than any other coxibs. Intriguingly, these two functions are mediated by different moieties of the celecoxib molecule and can be separated. The author, as well as others, have generated and investigated analogs of celecoxib that retain only one of these two functions. One derivative, 2,5-dimethyl-celecoxib (DMC), which retains the antiproliferative and apoptosis-inducing function, but completely lacks the COX-2 inhibitory activity, is able to mimic faithfully all of the numerous antitumor effects of celecoxib that have been investigated so far, including reduction of neovascularization and inhibition of experimental tumor growth in various in vivo tumor models. In view of the controversy that has recently arisen regarding the life-threatening side effects of this class of coxibs, it may be worthwhile to pursue further the potential benefits of drugs such as DMC for anticancer therapy. Because DMC is not a coxib yet potently maintains celecoxib's antitumor potential, one may be inclined to speculate that this novel compound could potentially be advantageous in the management of COX-2-independent cancers. In this summary, the implications of recent findings with DMC will be presented and discussed.
\end{abstract}

\section{KEY WORDS • 2,5-dimethyl-celecoxib • nonsteroidal antiinflammatory drug • glioma • celecoxib - Celebrex - cyclooxygenase}

A large body of data has indicated a role for NSAIDs in the prevention of various types of cancer. The biochemical mechanism generally ascribed to this effect is the inhibition of COX enzymes. Traditional NSAIDs, such as flurbiprofen, indomethacin, or sulindac, are able to inhibit COX-1 as well as COX-2, whereas newgeneration drugs, the so-called coxibs such as celecoxib (Celebrex), valdecoxib (Bextra), and rofecoxib (Vioxx), selectively inhibit only COX-2. Both enzymes, COX-1 and COX-2, catalyze the rate-limiting initial step in the synthesis of prostaglandins from the substrate arachidonic acid. ${ }^{44,84,114}$

The expression of COX-1 is constitutive in most tissues and is important for the maintenance of homeostatic func-

\footnotetext{
Abbreviations used in this paper: $\mathrm{CDK}=$ cyclin-dependent kinase; $\mathrm{COX}=$ cyclooxygenase; $\mathrm{DMC}=2,5$-dimethyl-celecoxib; ER-Ca ${ }^{++}$-ATPase $=$endoplasmic reticulum calcium adenosine triphosphatase; GBM = glioblastoma multiforme; MAPK/ERK = mitogen-activated protein kinase/extracellular signal-regulated kinase MEK = MAPK/ERK kinase; NF- $\mathrm{BB}=$ nuclear factor- $\mathrm{kB}$; NSAID = nonsteroidal antiinflammatory drug; PDK1 = 3-phosphoinositide-dependent protein kinase-1; $\mathrm{PGE}_{2}=$ prostaglandin $\mathrm{E}_{2}$; $\mathrm{PKB}=$ protein kinase $\mathrm{B}$.
}

tion. In contrast, COX-2 is an inducible isozyme that is strongly upregulated during pathological conditions such as inflammation and cancer. The $C O X$ gene is responsive to stimulation by growth factors, proinflammatory cytokines, and tumor promoters. ${ }^{104,118}$ In addition, many different types of human tumors have been shown to express highly elevated levels of COX-2, and it is thought that the increased expression of this enzyme might contribute to the carcinogenic process..$^{98,109,117}$

The disadvantage of selecting traditional COX inhibitors for clinical use is that they produce significant side effects in the patient, such as erosion and ulceration of the gastric mucosa. It is generally accepted that this is due to the inhibition of COX-1, which is considered a housekeeping enzyme and is therefore necessary for proper maintenance of mucosal integrity. Thus, in an attempt to avoid the drawbacks of traditional NSAIDs, new chemical variants were developed that selectively inhibit only COX2 and not COX-1. The premise behind these coxibs was the therapeutic benefit of traditional NSAIDs with fewer associated side effects; their development therefore generated great excitement for the potential advancement of cancer therapy. ${ }^{20,43,54,73}$

As the use of coxibs has greatly expanded during the past few years, however, it has become apparent that these 
drugs are not free from side effects. Instead, the long-term use of coxibs at high dosages, which has been believed to be necessary if used in anticancer therapy, is troubled by severe, potentially life-threatening risks such as cardiovascular events, renal injury, and gastrointestinal toxicity. ${ }^{6}, 46$, $81,97,105$ Because these unwanted effects are believed to be a class effect caused by the inhibition of COX-2 and the resulting imbalance of prostanoids, ${ }^{2,26,34}$ the initial euphoria regarding the potential antitumor benefits of these coxibs has vanished together with huge amounts of shareholder value. In addition, Vioxx was temporarily, and Bextra permanently, removed from the US market, and the Food and Drug Administration requested that labeling for all NSAIDs, including Celebrex, be revised to include a "boxed" or serious warning to highlight potentially lifethreatening risks associated with their use. ${ }^{17}$

Several studies are currently underway to more precisely assess the potential benefits and drawbacks of coxib use in the clinical setting, and it is expected that it will take several years before the final verdict is in on this controversial issue. In the meantime, a new area of research has been expanding that focuses on the initially surprising finding that at least some antitumor effects of NSAIDs do not appear to involve the inhibition of COXs. It has been speculated that, if one could harness these COX-independent antitumor effects and apply them in the clinical setting, it might be possible to avoid, or at least greatly reduce, the unwanted side effects that are caused by an imbalance of prostanoid levels. One compound with such allure is DMC, a close structural analog of celecoxib that completely lacks the COX-2-inhibitory function yet maintains potent antitumor activity both in vitro and in vivo.

\section{Introducing 2,5-Dimethyl-Celecoxib}

The compound DMC was first developed in the laboratory of Dr. Ching-Shih Chen at The Ohio State University (Columbus, OH). ${ }^{134}$ The basis for its discovery was provided by initial observations, ${ }^{41,48,61,106,129}$ which were later expanded, ${ }^{53,119}$ that celecoxib (Celebrex) was unique among the NSAIDs/coxibs because, in addition to its COX-2-inhibitory effect, it was able to potently induce tumor-cell apoptosis in the absence of any involvement of COX-2. Although the COX-independent effects of traditional NSAIDs had occasionally been reported, the second function of celecoxib was unique because it took place at relatively low drug concentrations, that is, celecoxib was able to inhibit proliferation and induce apoptosis in tumor cells in vitro at much lower concentrations than other coxibs (rofecoxib, valdecoxib, NS-398, and DuP697) or traditional NSAIDs (sulindac, flurbiprofen, indomethacin, among others). $47,48,51,53,70,106,134$ Most importantly, it was realized that the two activities of celecoxib-inhibition of COX-2 and suppression of cell proliferation-appeared to reside in different domains of the celecoxib molecule and therefore could be separated. ${ }^{106,134}$ From this insight arose the development of DMC, an analog of celecoxib that has lost the first function (the inhibition of COX-2) yet retained the second characteristic (the potent ability to inhibit cell proliferation and induce apoptosis; see Table 1).

Celecoxib is a 1,5-diaryl-substituted pyrazole and is chemically designated as 4-[5-(4-methylphenyl)-3-(trifluoromethyl)-1H-pyrazol-1-yl] benzenesulfonamide. ${ }^{88} \mathrm{~A}$
TABLE 1

A comparison of drug characteristics of celecoxib and DMC

\begin{tabular}{lcc}
\hline \multicolumn{1}{c}{ Activity } & Celecoxib & DMC \\
\hline inhibition of COX-2 & yes & no \\
induction of apoptosis & yes & yes \\
inhibition of proliferation & yes & yes \\
inhibition of tumor growth in vivo & yes & yes \\
inhibition of angiogenesis & yes & yes \\
\hline
\end{tabular}

close structural analog to celecoxib, DMC is designated as 4-[5-(2,5-dimethylphenyl)-3-(trifluoromethyl)-1H-pyrazol-1-yl] benzenesulfonamide. ${ }^{134}$ Whereas celecoxib has a methyl group at position 4 of the terminal phenyl ring, DMC has a 2,5-dimethyl moiety instead; otherwise, the remaining positions of their complex chemical structures are identical (Fig. 1). Details of the chemical synthesis of DMC have been provided elsewhere..$^{53}$

Initially, DMC's lack of COX-2-inhibitory activity was inferred from the characteristics of very similar compounds analyzed by Penning and associates. ${ }^{88}$ Subsequently, we showed that treatment of COX-2-overexpressing GBM cells with celecoxib, rofecoxib, or valdecoxib in vitro resulted in greatly reduced basal levels of $\mathrm{PGE}_{2}$, which was not the case when cells were exposed to DMC; 53 similarly, celecoxib, but not DMC, was able to prevent the increase in $\mathrm{PGE}_{2}$ production in A549 lung adenocarcinoma cells stimulated with interleukin-1.4 Although these observations were in agreement with a lack of COX-2 inhibitory activity, the inability of DMC to block the enzymatic activity of COX-2 has also been confirmed more directly in conventional in vitro COX-2 inhibitor assays, as shown in Fig. 2.

As determined from the crystal structure of the celecoxib-COX-2 complex, the interaction of celecoxib with COX-2 accommodates its single methyl group, which fits well into the NSAID binding space of the enzyme. ${ }^{35,90}$ In contrast, the two methyl groups in DMC at positions 2 and 5 of the phenyl ring presumably make it too bulky to fit in this space. In view of the analysis of very similar compounds by Penning and associates, ${ }^{88}$ one would conclude that DMC's lack of COX-2-inhibitory activity appears to be based primarily on steric hindrance, which prevents close interaction with the enzyme. For the same reason, the major oxidative metabolite of DMC that is extracted from serum, its dicarboxylate counterpart ${ }^{60}$ is not expected to exert any COX-2-inhibitory activity either.

Taken together, various approaches have established DMC as a COX-2-inactive analog of celecoxib. Amazing-

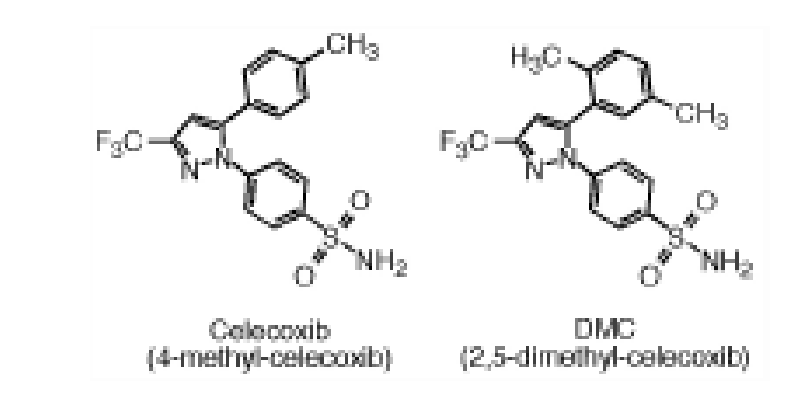

FIG. 1. Chemical structures of celecoxib and DMC. 


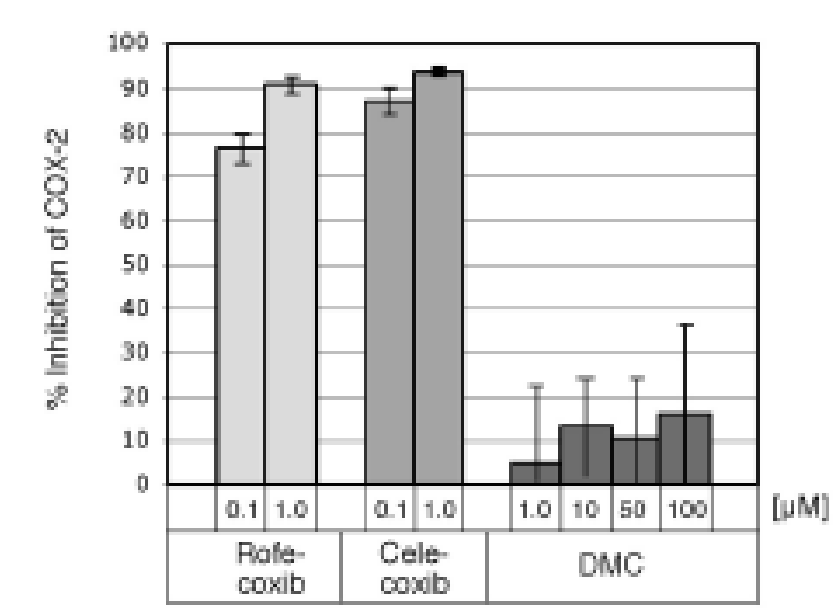

FIG. 2. Bar graph demonstrating inhibition of COX-2 activity due to various drugs. The ability of rofecoxib, celecoxib, and DMC to inhibit recombinant human $\mathrm{COX}-2$ was determined by performing the COX inhibitor screening assay (Cayman Chemicals, Ann Arbor, MI). As shown, $1 \mu \mathrm{M}$ rofecoxib or $1 \mu \mathrm{M}$ celecoxib inhibits the enzymatic activity of COX-2 by more than $90 \%$. In contrast, DMC has only a weak effect, even at substantially higher concentrations.

ly, however, as I will outline later in greater detail, DMC is able to mimic faithfully all of celecoxib's inhibitory effects on intracellular mitogenic and survival pathways, on cell proliferation in vitro, and on angiogenesis and tumor growth in vivo. ${ }^{4,52,53,60,70,106,134}$

\section{The COX-2-Independent Effects of NSAIDs}

Although there is clear evidence that at least some of the antitumor effects of NSAIDs may be mediated by the inhibition of COXs, ${ }^{18,74}$ there is also accumulating evidence that NSAIDs, including coxibs, are able to suppress tumor-cell growth and proliferation in the absence of any apparent involvement of either COX-1 or COX-2. A few examples of the latter follow. 1) There is no difference in the sensitivity of tumor cells harboring high levels of COX-2, low levels of COX-2, or no COX-2 at all (from knock-out mice) to the antiproliferative effects of coxibs in vitro or in vivo. ${ }^{51,53,110,119,129,132}$ 2) Low concentrations of coxibs in cell culture (sufficient to inhibit COX-2 fully) do not inhibit cell proliferation; rather, much higher concentrations are needed. . $^{, 51-53,79,85,119,129} 3$ ) In all instances investigated so far, DMC (which does not inhibit COX-2) faithfully mimics all antiproliferative (in vitro) and antitumorigenic (in vivo) effects of celecoxib.,52,53,60,70,106,134 4) Several studies, including ours, in which different concentrations of celecoxib were administered to tumor-bearing animals, found that generally $1000 \mathrm{ppm}$ of the drug (which results in serum concentrations of approximately $10 \mu \mathrm{M})^{60}$ is required to generate antitumor effects in vivo..$^{3,60,75,85}$, $115,124,135$ In contrast, $250 \mathrm{ppm}$ of celecoxib, which is sufficient to reduce greatly the $\mathrm{PGE}_{2}$ levels circulating in the blood of these animals, ${ }^{23}$ does not generally exert significant antitumor efficacy by itself ${ }^{23,60}$ (although head and neck tumor xenografts perhaps constitute a notable exception in this regard). ${ }^{135}$ Additionally, one study in which PC3 prostate carcinoma xenografts were used in nude mice demonstrated that DMC reduces experimental tumor growth more potently than celecoxib (even though the average serum concentrations of DMC were somewhat lower than those of celecoxib). ${ }^{60}$ Taken together, these data indicate that non-COX-2 targets must play an important role in the antitumor effects of celecoxib, and these additional targets appear to be affected only at drug concentrations higher than that necessary to inhibit COX-2.

Intriguingly, a study by Davis and colleagues ${ }^{23}$ showed that COX-2-inhibitory concentrations of celecoxib in vivo (250 ppm), although not antitumorigenic by themselves, were sufficient to enhance the antitumor effects of ionizing radiation on colon tumors grown in the footpad of mice; this cooperative effect was dependent on the presence of COX-2 in the tumor vasculature but not in the tumor cells themselves. From this finding, the authors concluded that celecoxib increases the radiosensitivity of established tumors in vivo through the inhibition of COX2 in endothelial cells. Although the findings of this study clearly indicate a role for COX-2 under these special conditions, and at the same time emphasize the importance of tumor angiogenesis, they also reveal that low COX2-inhibitory concentrations of celecoxib by themselves are not sufficient to reduce tumor growth but cooperate with additional intervention. Similarly, numerous other studies, in which mouse xenograft tumor models have been used, have been performed to explore whether the antitumor effects of conventional chemotherapy can be enhanced when combined with celecoxib. Many encouragingly positive results have been obtained, although most reports did not reveal whether the observed enhancing antitumor effect of celecoxib involved COX-2. ${ }^{14,97,100,115,124}$ From our own experience, we suspect that the antitumor effect of celecoxib in mouse xenograft tumor models will be "dominant" at high concentrations; in other words, when celecoxib is administered at sufficiently high concentrations ( $\geq 1000 \mathrm{ppm}$ ), it will exert antitumor and chemosensitizing effects without the requirement for COX-2 involvement.

Because of certain idiosyncrasies in mouse xenograft tumor models, it remains to be established whether such COX-2-independent antitumor effects of celecoxib take place in humans as well. Although numerous completed and ongoing clinical trials have demonstrated mixed outcomes with regard to celecoxib's potential to synergize with other cancer treatment modalities, it remains unclear whether, in these cases, the occasional successful effect of this drug involved the inhibition of COX-2. ${ }^{19,27,29,39,68,80,83,126}$ I propose that once DMC has been prepared for use in the clinical setting, its use can help clarify this issue.

\section{Non-COX-2 Targets of Celecoxib and DMC}

The experimental use of DMC has provided additional strong support for the claim that at least some of celecoxib's observed antitumor effects are independent of COX-2. In several different reports from the laboratory of Dr. Ching-Shih Chen (The Ohio State University) and from my own, DMC was shown to be at least as potent, if not more so, than celecoxib in suppressing tumor growth in various mouse xenograft tumor models ${ }^{52,53,60}$ (unpublished data). In all these cases, which included pancreatic carcinoma, Burkitt lymphoma, GBM, and multidrug-resistant 
multiple myeloma, celecoxib and DMC were able to suppress tumor formation significantly. Moreover, it has been demonstrated that both drugs displayed potent antiangiogenic activity. ${ }^{70}$ Because DMC does not inhibit COX-2, this line of evidence strongly suggests that inhibition of COX-2 is not required for these antitumor and antiangiogenic effects to take place. This conclusion, of course, begs the question as to the cellular components and mechanisms that partake in and mediate the COX-2-independent effects of these two drugs.

The activities of several intracellular signaling and cellcycle molecules have been found to be affected by treatment of cells with celecoxib. $30,42,45,48,51,64,113,133,134$ Although it could be argued that perhaps some cellular components are indirectly affected, that is, as a consequence of the inhibition of COX-2, most of these drug effects are also observed in COX-2-negative cells and are replicated by DMC, which does not inhibit COX-2; these findings indicate that COX-2 indeed is not involved. The following intracellular molecules, all of which have critical roles in tumor cell proliferation and survival, have been found to be similarly affected by treatment of cells with celecoxib and DMC.

From among the non-COX-2 targets of celecoxib, one protein has been proposed to act as a receptor for celecoxib: PDK1., ${ }^{3,60}$ Because celecoxib was shown to inhibit the enzymatic activity of purified PDK1 directly in the test tube, it was concluded that this protein kinase might be the major non-COX-2 receptor of celecoxib that mediates the COX-2-independent effects of the drug. ${ }^{3,60}$ In cells, PDK1 activates the PKB/Akt survival pathway by directly phosphorylating its in vivo substrate Akt/PKB; accordingly, it has been shown that treatment of cells with celecoxib results in decreased levels of Akt/PKB phosphorylation. ${ }^{3,60}$ This discovery seems to provide a reasonable explanation for the COX-2-independent antitumor effects of celecoxib: when PDK1 is inhibited by celecoxib, the activity of the cell-survival kinase Akt/PKB is downregulated and, therefore, cells are more prone to apoptosis. In further support of this view, the activity of PDK1 has been found to be inhibited by DMC as well. ${ }^{60}$

Subsequent studies in our laboratory, however, have not yielded findings supporting the aforementioned supposition. For example, we have been unable to replicate the reported inhibition of purified PDK1 by celecoxib or DMC in vitro. ${ }^{53}$ Furthermore, the downregulation of Akt/ PKB phosphorylation by celecoxib and DMC appears to be highly cell-type specific and is often only achieved under conditions of serum deprivation in the culture medi- um. Although detected in some cell types, $3,45,64,106$ this downregulation of $\mathrm{AKt} / \mathrm{PKB}$ has not been observed by others $;{ }^{14}$ in our own studies, we were unable to detect drug-induced changes in $\mathrm{Akt} / \mathrm{PKB}$ phosphorylation in cases of Burkitt lymphoma, multiple myeloma, and various GBM cell lines - even though the proliferation of all these cells was potently inhibited by celecoxib and DMC ${ }^{52,53}$ Moreover, we found that mouse embryonic stem cells with knocked-out PDK1 alleles (PDK1- cells) ${ }^{125}$ were as sensitive to celecoxib and DMC as their wild-type counterparts; that is, the extent of growth inhibition by these drugs was not affected by the absence of PDK1 in these cells (unpublished data). Taken together, the function of PDK1 as a major receptor for celecoxib and DMC remains somewhat controversial, and more experiments are needed to clarify this issue.

Other important cellular components found to be affected by celecoxib and DMC are the cyclins, which are the essential regulatory subunits of CDKs. Cyclin-dependent kinase activity, also called the cell-cycle engine, is required for cell proliferation to take place; without it, cells are arrested in the cell cycle. ${ }^{40,82}$ Work produced in our laboratory has demonstrated that celecoxib and DMC block the transcription of cyclins $\mathrm{A}$ and $\mathrm{B}$, thus preventing the synthesis of these two critical CDK subunits. ${ }^{51,53}$ As a result, drug-treated cells lose CDK activity and are unable to proliferate. Similar to observations regarding other effects caused by these two drugs, other coxibs and traditional NSAIDs do not affect the expression of these cyclins, downregulate CDK activity, or inhibit cell proliferation at comparable concentrations. ${ }^{7,41,51,79,85}$ Because celecoxib and DMC consistently inhibit the expression of cyclins in different tumor cell lines, and in consideration of the indispensable role cyclins play in the regulation of cell proliferation, it is reasonable to conclude that these targets may play a major role in mediating the antitumor effects of these drugs.

In addition to cyclins and PDK1, there are other cellular components that have been found to be affected by celecoxib and DMC (see Fig. 3). Exemplary reports of coxib/DMC effects on these targets include the following:

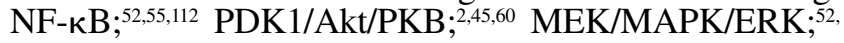
${ }_{53,106,134}$ CDKs/cyclins, ${ }^{52,53,70}$ ER-Ca ${ }^{++}$-ATPase, ${ }^{47,112}$ carbonic anhydrase ${ }^{57,120}$ survivin; ${ }^{10,52}$ and the caspases. ${ }^{10,25,52}$ Although details of these effects have not yet been established (it is currently unknown how these drugs impinge on the activity of the cellular components), the components all constitute proteins that are critically important for cell-growth regulation and survival or apoptosis. Ad-

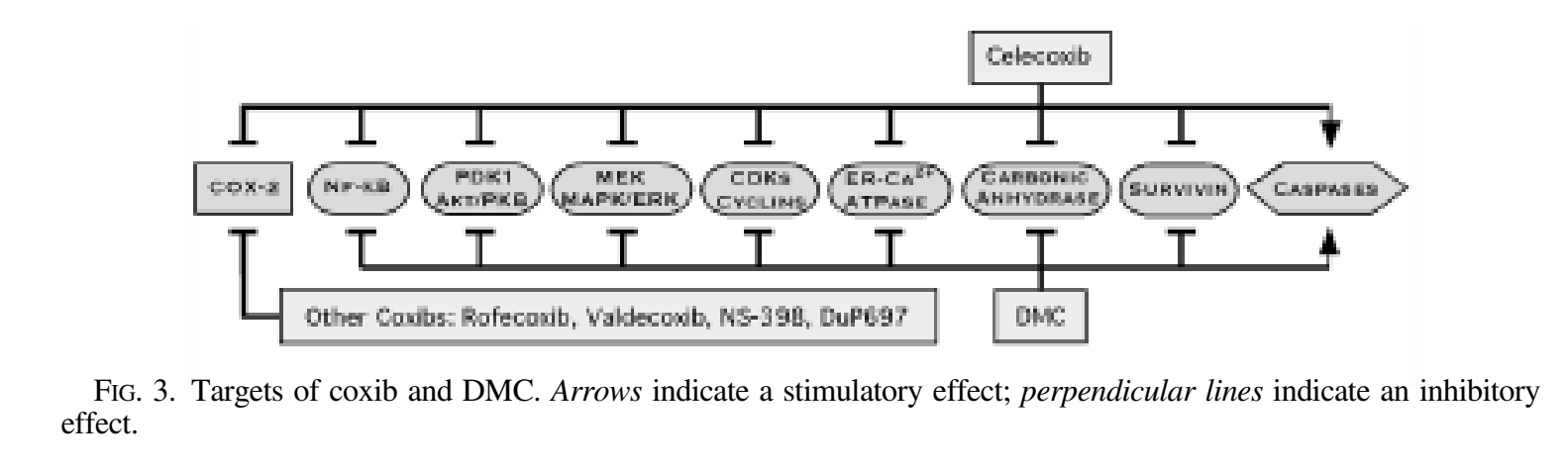


ditional studies are needed to determine the role and precise contribution of these targets to the antiproliferative and apoptosis-inducing processes initiated by celecoxib and DMC. In this regard, it should be noted that the apparent multitarget nature of these two drugs could be beneficial for purposes of anticancer therapy. As it has emerged that multiple pathways in tumor cells should be targeted to achieve therapeutic success, recently published views presenting efficient cancer treatments favor multimodal therapies and combination drug treatments. ${ }^{28,31-33,101}$ Within this context, the multifaceted molecular consequences of tumor cell treatment with celecoxib and DMC may actually be highly desirable, and may be beneficial for treatment of many different types of tumors; this has indeed been indicated by findings of numerous studies of the antitumor effects of celecoxib and DMC in a great variety of different tumor cells in vitro and in vivo (some of these studies

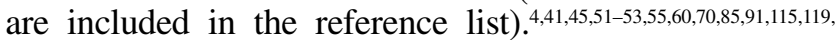
124,129

\section{The Conundrum of In Vitro Versus In Vivo Effects}

It has been proposed that the COX-2-independent effects of celecoxib might be an in vitro artifact and do not occur in vivo. ${ }^{94,124}$ This view is based on the observation that there is a substantial difference between effective drug concentrations used in vitro and those achieved in the serum of patients. For example, it generally requires between 10 and $75 \mu \mathrm{M}$ of celecoxib or DMC to block proliferation and induce apoptosis of tumor cells in vitro (in cell culture), whereas the serum concentration of celecox$\mathrm{ib}$ in patients or experimental animals ranges from 1 to 10 $\mu \mathrm{M}^{22,86}$ 2,5-dimethyl-celecoxib appears to have similar pharmacokinetics and can reach $10 \mu \mathrm{M}$ in the serum of laboratory mice. ${ }^{60} \mathrm{In}$ addition to these discrepancies in effective drug concentrations, it has been suggested that celecoxib's antitumor effect can be mediated via the inhibition of COX-2 solely in endothelial cells: tumor inhibition then is achieved via the blockage of angiogenesis, independent of the COX-2 status of the tumor cells themselves. ${ }^{23,124}$

Several recent findings appear to oppose the aforementioned arguments. For example, DMC, which does not affect COX-2 at all, potently mimics the antitumor effects of celecoxib in tumor-bearing animals..$^{52,53,60}$ In addition, DMC (similar to celecoxib) has been shown to block angiogenesis in the chicken chorioallantoic membrane model. ${ }^{70}$ Thus, although these results do not lead us to question the well-established decisive role of COX-2 in tumor angiogenesis, ${ }^{37,123,124,135}$ they do suggest that celecox$\mathrm{ib}$ and DMC do not require the inhibition of COX-2 to exert their antitumor and antiangiogenic effects-at least not in the in vivo models used thus far.

In culture the inhibition of COX-2 in cells has no inhibitory effect on their proliferation or survival, and cells lacking the $C O X-2$ (and/or $C O X-1$ ) gene proliferate normally and exhibit comparable sensitivity to NSAIDinduced growth inhibition and cell death. ${ }^{132}$ In contrast, some of the non-COX-2 targets of celecoxib and DMC, for example, cyclins A and B, are vital for cell proliferation to take place. Therefore, the presence of certain nonCOX-2 targets could very well suffice to explain these drugs' growth-inhibitory action-if drug effects on such targets can be verified in vivo as well. Remarkably, this latter fundamental condition has recently been met.

It has been demonstrated that some non-COX-2 targets of celecoxib and DMC, which are affected at relatively high drug concentrations in cell culture in vitro, are similarly targeted in xenograft tumor models in vivo, in which substantially lower drug concentrations are present. For example, the reduced growth of a human Burkitt lymphoma in animals treated with celecoxib or DMC closely correlated with substantial (approximately 80-90\%) downregulation of cyclins A and B expression. ${ }^{53}$ Similarly, inhibition of PDK1 in a pancreatic carcinoma xenograft mouse model correlated with reduced tumor growth in response to treatment of these animals with DMC. ${ }^{60}$

An additional target of celecoxib and DMC is survivin, which belongs to the family of antiapoptotic proteins. ${ }^{1,66}$ Expression of survivin is frequently found to be greatly elevated in tumor tissue and is thought to confer resistance to chemo- and radiotherapy on these cells. For example, findings of several studies have demonstrated that the experimentally induced decrease in survivin expression (for example, that due to antisense or small interfering RNA approaches ${ }^{16}$ ) causes greatly enhanced apoptosis of these cells in response to treatment with various anticancer modalities. ${ }^{8,13,72,87,96,127,130}$ In this regard, our most recent studies have provided evidence that celecoxib and DMC are able to block survivin expression completely in vitro and in vivo, concomitantly stimulate apoptosis, and substantially increase the chemosensitivity of tumor cells to certain anticancer drugs (Pyrko, et al., unpublished data). Considering the critical function of survivin as a custodian of tumor-cell survival, these results suggest that this protein may play an important role in the apoptosis-inducing mechanisms affected by celecoxib and DMC. In this regard, I further speculate that these processes could also participate in the previously described radiosensitization effects of celecoxib in GBM cells, ${ }^{56,89,93}$ although this has not yet been investigated using DMC.

Together, the aforementioned results clearly demonstrate that similar molecular and cellular responses can be achieved in vitro and in vivo, even though the required drug concentrations appear to differ. The basis for the concentration issue has remained unclear, although it should be noted that effective drug concentrations in vitro can be lowered substantially under altered cell-culture conditions; in particular, a reduction in the serum concentrations of cell-culture medium, from the conventional 10 to $2 \%$ or lower, will dramatically decrease the median inhibition concentration of celecoxib or DMC required to induce growth arrest and apoptosis. ${ }^{48,60,134}$

In any case, the aforementioned examples thoroughly demonstrate that celecoxib and DMC are able to impinge potently on non-COX-2 targets in vivo as well, and suggest that at least some of these processes can serve as a rational explanation for the reported COX-2-independent antitumor effects of these drugs.

\section{Implications for Glioma Therapy}

The typical intervention used in patients harboring malignant gliomas consists of resection followed by radiotherapy and then chemotherapy. ${ }^{5}$ Despite this enormous tripartite effort, however, the median survival period for a 
patient with a newly diagnosed GBM is frustratingly short. ${ }^{69}$ In addition, although radiation treatment after surgery clearly results in benefits for survival, a major complicating factor is the significant neurotoxic side effects, which are prominent, particularly in the older population, and severely reduce the quality of life for affected patients. ${ }^{62,116,121}$ In this regard, novel therapeutic approaches, such as stereotactic radiosurgery or the inclusion of radiation sensitizers, may hold some promise for an improved benefit/harm ratio. ${ }^{107,122,131}$

Not surprisingly, after celecoxib burst onto the stage of anticancer research, numerous groups began to evaluate its potential for inclusion in brain-tumor therapy. The underlying rationale was based on the earlier observation that expression of COX-2, as well as levels of its reaction products, the prostaglandins, is frequently found to be elevated in cases of glioma..$^{9,24,49,58}$ In addition, a high level of COX2 expression is associated with clinically more aggressive tumors, such as GBM, and is a strong predictor of poor survival. ${ }^{103}$ It has therefore been postulated that inhibition of COX-2 activity in such cells might be beneficial for management of these tumors. ${ }^{15}$

Studies by my group and others have established that celecoxib is able to potently inhibit proliferation and invasion of several GBM cell lines in vitro, but this effect takes place independently of COX-2. ${ }^{51,63}$ Using an orthotopic rat gliosarcoma model, Nam, et al., ${ }^{78}$ found that celecoxib, administered orally, significantly reduced the incidence and size of tumors, and that tumor cells from treated rats had lower levels of phospho-Akt/PKB, a previously identified alternative target (via the inhibition of PDK1; see earlier discussion) of this drug. Using a similar orthotopic rattumor model, this same group also reported ${ }^{50}$ that combination therapy of celecoxib and temozolomide, a methylating agent, ${ }^{108}$ resulted in significantly reduced tumor volume and microvessel density compared with untreated controls or single-agent therapy. In our own in vivo studies, we found that celecoxib and DMC were able to inhibit potently the growth of human GBM cells in a xenograft nude mouse model (Pyrko, et al., unpublished data). In this case, the inhibition of tumor growth coincided with increased tumor-cell apoptosis and with decreased expression of survivin in the tumor tissue. This latter observation is particularly interesting because survivin levels in human GBM have been associated with increased tumor-cell survival and progression to a more aggressive tumor phenotype. ${ }^{12,13,21,128}$ Taken together, the above-referenced reports support the idea that celecoxib may be beneficial as part of GBM therapy, but that these effects may also take place independently of the involvement of COX -2 and, thus, that perhaps DMC should be further evaluated as well.

Several ongoing and completed clinical trials have been performed to evaluate the benefit of including celecoxib in glioma chemotherapy. For example, the results of a Phase II trial of irinotecan (CPT-11), a topoisomerase I inhibitor, ${ }^{36}$ in combination with celecoxib showed encouraging news about this regimen in heavily pretreated patients with recurrent malignant glioma. ${ }^{95}$ In contrast, the combination of celecoxib and 13-cis-retinoic acid was not more effective than 13-cis-retinoic acid alone in the treatment of recurrent GBM. ${ }^{65}$ Additional Phase II trials are currently underway to investigate the potential benefit of celecoxib in combination with other chemotherapeutic or antiangiogenic drugs, such as temozolomide, thalidomide, isotretinoin, etoposide, or cyclophosphamide, in patients who have undergone radiotherapy for GBM. Although the results of these trials will provide important insights into the potential usefulness of celecoxib as part of combination chemotherapy, they will not answer the question of whether inhibition of COX2 is required for such effects (if any).

In addition to combination chemotherapy, celecoxib is also being evaluated as a radiosensitizer, and in this regard, there is strong evidence that the inhibition of COX-2 may play an important role. ${ }^{15,76,77}$ For example, various traditional NSAIDs, as well as selective COX-2 inhibitors, have been shown to enhance intrinsic tumor cell radiosensitivity in vitro. ${ }^{56,89,92}$ This effect was also exerted by celecoxib and other selective COX-2 inhibitors in various animal tumor models in vivo. ${ }^{23,56,71,89,92}$ Two reports have indicated that the radiosensitizing effects of celecoxib preferentially took place in tumor cells with elevated levels of COX-2,, ${ }^{92,102}$ a finding that is in stark contrast to celecoxib's antitumor effects in monotherapy, in which the COX-2 status of tumor cells has no relevance for the efficacy of druginduced antigrowth effects (see earlier discussion). It should be noted, however, that the radiosensitizing effects of this drug have also been observed in experimental tumors in which COX-2 expression was restricted to the tumor neovasculature. ${ }^{23}$ As already mentioned, this particular study is quite intriguing because it demonstrates that celecoxib alone, at low dosages (250 ppm), does not inhibit tumor growth, but does cause radiosensitization. It is quite likely that this result reflects the dual features of celecoxib - the inhibition of COX-2 and the ability to stimulate apoptosis - and indicates that for purposes of radiosensitization, the effect of celecoxib on COX-2 activity might be the decisive attribute.

Unlike lung cancer research in which clinical studies into the radiosensitizing effects of celecoxib are more advanced, ${ }^{38,59}$ literature regarding such an effect on brain cancer is scarce. One notable exception is a Phase I/II study of patients with unresectable brain metastases, in which it was found that radiotherapy plus celecoxib is safe and possibly useful for these patients. ${ }^{11}$ Thus, although the findings of some studies are encouraging and suggest a role for celecoxib as a radiosensitizer in the clinical setting, only time (and more clinical trials) will tell whether this promise indeed will hold up.

With regard to the involvement of COX-2, it appears that this enzyme may be the primary target of celecoxib when it comes to radiosensitization. Nevertheless, other cellular components, such as PDK1-Akt, PKB, and survivin, known targets of celecoxib, are major players in cellular radioprotection as well. ${ }^{13,67,72,96,99}$ Therefore, it may be desirable to determine the ability (or lack thereof) of DMC to induce radiosensitization of tumor cells. Should we find that DMC is unable to increase radiosensitivity, this would provide very solid support for the critical role of COX-2 in this process. In the meantime, I will postulate that celecoxib's COX-2 inhibitory function is probably important for the radiosensitization of tumor cells, but perhaps is not generally required in instances of chemosensitization or for the drug's antitumor effects in monotherapy. Because it is unclear what the beneficial effects of COX-2 inhibition may be in treating gliomas, the use of DMC - without the potential side effects of COX-2 inhibition, but with the antiapoptotic properties of celecoxib-may prove beneficial in combination with conventional glioma therapy. 


\section{Conclusions}

Although DMC is unable to inhibit COX-2, it nevertheless accurately and potently mimics all of celecoxib's antitumor effects investigated so far. This congruence extends to in vitro and in vivo studies and includes experiments performed in various xenograft mouse tumor models. These results strongly argue against a role of COX-2 in these processes and, consequently, several other intracellular key regulatory components have been identified that could serve to explain the observed drug effects. For example, the essential cell-cycle regulatory cyclins or the important cell-survival custodians PDK1 and survivin present candidates for major players that could mediate the antitumor effects of celecoxib and DMC. In addition, the possibility exists that several of these alternative targets must cooperate to promote the full drug effects and, thus, celecoxib and DMC exert their pleiotropic effects via multiple targets.

Although the work with DMC has contributed important insights into the underlying mechanisms of the wellrecognized COX-2-independent antitumor effects of celecoxib, it has also generated exciting speculation regarding its potential for future clinical use. Because the recently recognized life-threatening side effects of long-term clinical coxib use have been linked to an imbalance in prostanoid levels, one would expect that DMC, due to its inability to inhibit COX-2, would not cause similar side effects and, perhaps, could be used at even higher concentrations. Thus, at least in those cancers in which the inhibition of COX-2 appears negligible, it might be worthwhile to evaluate DMC's potential as a noncoxib alternative for anticancer purposes.

\section{Acknowledgments}

I thank all members of the GReAT (Glioma Research Activity Team) at USC for their valuable input, and I express my gratitude to Florence M. Hofman, Ph.D. (Department of Pathology, USC), Nicos A. Petasis, Ph.D. (Department of Chemistry, USC), and Thomas C. Chen, M.D., Ph.D. (Department of Neurosurgery, USC) for constructive discussions and productive collaborations. I acknowledge Ching-Shih Chen, Ph.D. (The Ohio State University, Columbus, $\mathrm{OH}$ ), for providing a sample of DMC for preliminary experiments, which stimulated subsequent projects in my laboratory.

\section{References}

1. Altieri DC: Survivin, versatile modulation of cell division and apoptosis in cancer. Oncogene 22:8581-8589, 2003

2. Anonymous: Editorial: Painful lessons. Nat Struct Mol Biol 12:205, 2005

3. Arico S, Pattingre S, Bauvy C, et al: Celecoxib induces apoptosis by inhibiting 3-phosphoinositide-dependent protein kinase1 activity in the human colon cancer HT-29 cell line. J Biol Chem 277:27613-27621, 2002

4. Backhus LM, Petasis NA, Uddin J, et al: Dimethyl celecoxib as a novel non-cyclooxygenase 2 therapy in the treatment of nonsmall cell lung cancer. J Thorac Cardiovasc Surg 130:1406-1412, 2005

5. Brandes AA: State-of-the-art treatment of high-grade brain tumors. Semin Oncol 30 (Suppl 19):4-9, 2003

6. Bresalier RS, Sandler RS, Quan H, et al: Cardiovascular events associated with rofecoxib in a colorectal adenoma chemoprevention trial. N Engl J Med 352:1092-1102, 2005
7. Buecher B, Bouancheau D, Broquet A, et al: Growth inhibitory effect of celecoxib and rofecoxib on human colorectal carcinoma cell lines. Anticancer Res 25:225-233, 2005

8. Cao C, Mu Y, Hallahan DE, et al: XIAP and survivin as therapeutic targets for radiation sensitization in preclinical models of lung cancer. Oncogene 23:7047-7052, 2004

9. Castelli MG, Chiabrando C, Fanelli R, et al: Prostaglandin and thromboxane synthesis by human intracranial tumors. Cancer Res 49:1505-1508, 1989

10. Catalano A, Graciotti L, Rinaldi L, et al: Preclinical evaluation of the nonsteroidal antiinflammatory agent celecoxib on malignant mesothelioma chemoprevention. Int J Cancer 109: 322-328, 2004

11. Cerchietti LCA, Bonomi MR, Navigante AH, et al: Phase I/II study of selective cyclooxygenase- 2 inhibitor celecoxib as a radiation sensitizer in patients with unresectable brain metastases. J Neurooncol 71:73-81, 2005

12. Chakravarti A, Noll E, Black PM, et al: Quantitatively determined survivin expression levels are of prognostic value in human gliomas. J Clin Oncol 20:1063-1068, 2002

13. Chakravarti A, Zhai GG, Zhang M, et al: Survivin enhances radiation resistance in primary human glioblastoma cells via caspase-independent mechanisms. Oncogene 23:7494-7506, 2004

14. Chen Z, Zhang X, Li M, et al: Simultaneously targeting epidermal growth factor receptor tyrosine kinase and cyclooxygenase-2, an efficient approach to inhibition of squamous cell carcinoma of the head and neck. Clin Cancer Res 10:5930-5939, 2004

15. Choy H, Milas L: Enhancing radiotherapy with cyclooxygenase-2 enzyme inhibitors: a rational advance? J Natl Cancer Inst 95:1440-1452, 2003

16. Coma S, Noe V, Lavarino C, et al: Use of siRNAs and antisense oligonucleotides against survivin RNA to inhibit steps leading to tumor angiogenesis. Oligonucleotides 14:100-113, 2004

17. Couzin J: FDA panel urges caution on many anti-inflammatory drugs. Science 307:1183-1184, 2005

18. Crosby CG, DuBois RN: The cyclooxygenase- 2 pathway as a target for treatment or prevention of cancer. Expert Opin Emerg Drugs 8:1-7, 2003

19. Csiki I, Morrow JD, Sandler A, et al: Targeting cyclooxygenase-2 in recurrent non-small cell lung cancer: a phase II trial of celecoxib and docetaxel. Clin Cancer Res 11:6634-6640, 2005

20. Dannenberg AJ, Subbaramaiah K: Targeting cyclooxygenase-2 in human neoplasia: rationale and promise. Cancer Cell 4: 431-436, 2003

21. Das A, Tan WL, Teo J, et al: Expression of survivin in primary glioblastomas. J Cancer Res Clin Oncol 128:302-306, 2002

22. Davies NM, McLachlan AJ, Day RO, et al: Clinical pharmacokinetics and pharmacodynamics of celecoxib: a selective cyclooxygenase-2 inhibitor. Clin Pharmacokinet 38:225-242, 2000

23. Davis TW, O'Neal JM, Pagel MD, et al: Synergy between celecoxib and radiotherapy results from inhibition of cyclooxygenase-2-derived prostaglandin $E_{2}$, a survival factor for tumor and associated vasculature. Cancer Res 64:279-285, 2004

24. Deininger MH, Weller M, Streffer J, et al: Patterns of cyclooxygenase-1 and -2 expression in human gliomas in vivo. Acta Neuropathol (Berl) 98:240-244, 1999

25. Ding H, Han C, Zhu J, et al: Celecoxib derivatives induce apoptosis via the disruption of mitochondrial membrane potential and activation of caspase 9. Int J Cancer 113:803-810, 2005

26. Drazen JM: COX-2 inhibitors-a lesson in unexpected problems. N Engl J Med 352: 1131-1132, 2005

27. Dy GK, Mandrekar S, Peethambaram PP, et al: A phase I trial of celecoxib in combination with docetaxel and irinotecan in patients with advanced cancer. Cancer Chemother Pharmacol 56:623-628, 2005

28. Eich HT, Hero B, Staar S, et al: Multimodality therapy includ- 
ing radiotherapy and chemotherapy improves event-free survival in stage $\mathrm{C}$ esthesioneuroblastoma. Strahlenther Onkol 179:233-240, 2003

29. El-Rayes BF, Zalupski MM, Shields AF, et al: A Phase II study of celecoxib, gemcitabine, and cisplatin in advanced pancreatic cancer. Invest New Drugs 23:583-590, 2005

30. Elder DJE, Halton DE, Playle LC, et al: The MEK/ERK pathway mediates COX-2-selective NSAID-induced apoptosis and induced COX-2 protein expression in colorectal carcinoma cells. Int J Cancer 99:323-327, 2002

31. Emens LA, Reilly RT, Jaffee EM: Cancer vaccines in combination with multimodality therapy. Cancer Treat Res 123: 227-245, 2005

32. Fang B, Roth JA: The role of gene therapy in combined modality treatment strategies for cancer. Curr Opin Mol Ther 5: 475-482, 2003

33. Farray D, Mirkovic N, Albain KS: Multimodality therapy for stage III non-small-cell lung cancer. J Clin Oncol 23: 3257-3269, 2005

34. Fitzgerald GA: Coxibs and cardiovascular disease. N Engl J Med 351:1709-1711, 2004

35. Flower RJ: The development of COX2 inhibitors. Nat Rev Drug Discov 2:179-191, 2003

36. Friedman HS, Keir ST, Houghton PJ: The emerging role of irinotecan (CPT-11) in the treatment of malignant glioma in brain tumors. Cancer 97:2359-2362, 2003

37. Gately S, Li WW: Multiple roles of COX-2 in tumor angiogenesis: a target for antiangiogenic therapy. Semin Oncol 31 (Suppl 7):2-11, 2004

38. Gore E: Celecoxib and radiation therapy in non-small-cell lung cancer. Oncology (Williston Park) 18 (Suppl 14):10-14, 2004

39. Govindan R, McLeod H, Mantravadi P, et al: Cisplatin, fluorouracil, celecoxib, and RT in resectable esophageal cancer: preliminary results. Oncology (Williston Park) 18 (Suppl 14): $18-21,2004$

40. Graña X, Reddy EP: Cell cycle control in mammalian cells: role of cyclins, cyclin dependent kinases (CDKs), growth suppressor genes and cyclin-dependent kinase inhibitors (CKIs). Oncogene 11:211-219, 1995

41. Grösch S, Tegeder I, Niederberger E, et al: COX-2 independent induction of cell cycle arrest and apoptosis in colon cancer cells by the selective COX-2 inhibitor celecoxib. FASEB J 15: 2742-2744, 2001

42. Gupta RA, DuBois RN: Colorectal cancer prevention and treatment by inhibition of cyclooxygenase-2. Nat Rev Cancer 1: 11-21, 2001

43. Hawk ET, Viner JL, Dannenberg A, et al: COX-2 in cancer-a player that's defining the rules. J Natl Cancer Inst 94: 545-546, 2002

44. Howe LR, Dannenberg AJ: A role for cyclooxygenase-2 inhibitors in the prevention and treatment of cancer. Semin Oncol 29 (Suppl 11):111-119, 2002

45. Hsu AL, Ching TT, Wang DS, et al: The cyclooxygenase-2 inhibitor celecoxib induces apoptosis by blocking Akt activation in human prostate cancer cells independently of Bcl-2. J Biol Chem 275:11397-11403, 2000

46. Jenkins JK: US Food and Drug Administration: Analysis and recommendations for Agency action regarding non-steroidal anti-inflammatory drugs and cardiovascular risk. J Pain Palliat Care Pharmacother 19:83-97, 2005

47. Johnson AJ, Hsu AL, Lin HP, et al: The cyclo-oxygenase-2 inhibitor celecoxib perturbs intracellular calcium by inhibiting endoplasmic reticulum $\mathrm{Ca}^{2+}$-ATPases: a plausible link with its anti-tumour effect and cardiovascular risks. Biochem J 366:831-837, 2002

48. Johnson AJ, Song X, Hsu A, et al: Apoptosis signaling pathways mediated by cyclooxygenase- 2 inhibitors in prostate cancer cells. Adv Enzyme Regul 41:221-235, 2001
49. Joki T, Heese O, Nikas DC, et al: Expression of cyclooxygenase 2 (COX-2) in human glioma and in vitro inhibition by a specific COX-2 inhibitor, NS-398. Cancer Res 60:4926-4931, 2000

50. Kang SG, Kim JS, Park K, et al: Combination celecoxib and temozolomide in C6 rat glioma orthotopic model. Oncol Rep 15:7-13, 2006

51. Kardosh A, Blumenthal M, Wang WJ, et al: Differential effects of selective COX-2 inhibitors on cell cycle regulation and proliferation of glioblastoma cell lines. Cancer Biol Ther 3: 55-62, 2004

52. Kardosh A, Soriano N, Liu YT, et al: Multitarget inhibition of drug-resistant multiple myeloma cell lines by dimethyl-celecoxib (DMC), a non-COX-2 inhibitory analog of celecoxib. Blood 106:4330-4338, 2005

53. Kardosh A, Wang W, Uddin J, et al: Dimethyl-celecoxib (DMC), a derivative of celecoxib that lacks cyclooxygenase-2inhibitory function, potently mimics the anti-tumor effects of celecoxib on Burkitt's lymphoma in vitro and in vivo. Cancer Biol Ther 4:571-582, 2005

54. Keller JJ, Giardiello FM: Chemopreventive strategies using NSAIDs and COX-2 inhibitors. Cancer Biol Ther 2 (Suppl 1): S140-S149, 2003

55. Kim SH, Song SH, Kim SG, et al: Celecoxib induces apoptosis in cervical cancer cells independent of cyclooxygenase using NF- $\mathrm{KB}$ as a possible target. J Cancer Res Clin Oncol 130:551-560, 2004

56. Kishi K, Petersen S, Petersen C, et al: Preferential enhancement of tumor radioresponse by a cyclooxygenase- 2 inhibitor. Cancer Res 60:1326-1331, 2000

57. Knudsen JF, Carlsson U, Hammarström P, et al: The cyclooxygenase-2 inhibitor celecoxib is a potent inhibitor of human carbonic anhydrase II. Inflammation 28:285-290, 2004

58. Kokoglu E, Tuter Y, Sandikci KS, et al: Prostaglandin E2 levels in human brain tumor tissues and arachidonic acid levels in the plasma membrane of human brain tumors. Cancer Lett 132:17-21, 1998

59. Komaki R, Liao Z, Milas L: Improvement strategies for molecular targeting: cyclooxygenase-2 inhibitors as radiosensitizers for non-small cell lung cancer. Semin Oncol 31 (Suppl 11): 47-53, 2004

60. Kulp SK, Yang YT, Hung CC, et al: 3-phosphoinositide-dependent protein kinase-1/Akt signaling represents a major cyclooxygenase-2-independent target for celecoxib in prostate cancer cells. Cancer Res 64:1444-1451, 2004

61. Kusunoki N, Yamazaki R, Kawai S: Induction of apoptosis in rheumatoid synovial fibroblasts by celecoxib, but not by other selective cyclooxygenase 2 inhibitors. Arthritis Rheum 46: 3159-3167, 2002

62. Laperriere N, Zuraw L, Cairncross G, et al: Radiotherapy for newly diagnosed malignant glioma in adults: a systematic review. Radiother Oncol 64:259-273, 2002

63. Lee HC, Park IC, Park MJ, et al: Sulindac and its metabolites inhibit invasion of glioblastoma cells via down-regulation of Akt/PKB and MMP-2. J Cell Biochem 94:597-610, 2005

64. Leng J, Han C, Demetris AJ, et al: Cyclooxygenase-2 promotes hepatocellular carcinoma cell growth through AKT activation: evidence for AKT inhibition in celecoxib-induced apoptosis. Hepatology 38:756-768, 2003

65. Levin VA, Giglio P, Puduvalli VK, et al: Combination chemotherapy with 13-cis-retinoic acid and celecoxib in the treatment of glioblastoma multiforme. J Neurooncol, 2005 [Epub ahead of print] http://www.springerlink.com/(a04p3z45ix2aj3r uiydm1345)/app/home/contribution.asp?referrer=parent\&backto =issue,84,103; journal,1,223; linkingpublicationresults, 1:102942,1

66. Li F: Survivin study: what is the next wave? J Cell Physiol 197: 8-29, 2003

67. Liang K, Jin W, Knuefermann C, et al: Targeting the phosphatidylinositol 3-kinase/Akt pathway for enhancing breast cancer cells to radiotherapy. Mol Cancer Ther 2:353-360, 2003 
68. Liao Z, Komaki R, Milas L, et al: A phase I clinical trial of thoracic radiotherapy and concurrent celecoxib for patients with unfavorable performance status inoperable/unresectable nonsmall cell lung cancer. Clin Cancer Res 11:3342-3348, 2005

69. Lin CL, Lieu AS, Lee KS, et al: The conditional probabilities of survival in patients with anaplastic astrocytoma or glioblastoma multiforme. Surg Neurol 60:402-406, 2003

70. Lin HP, Kulp SK, Tseng PH, et al: Growth inhibitory effects of celecoxib in human umbilical vein endothelial cells are mediated through $\mathrm{G}_{1}$ arrest via multiple signaling mechanisms. Mol Cancer Ther 3:1671-1680, 2004

71. Liu W, Chen Y, Wang W, et al: Combination of radiation and celebrex (celecoxib) reduce mammary and lung tumor growth. Am J Clin Oncol 26 (Suppl 2):S103-S109, 2003

72. Lu B, Mu Y, Cao C, et al: Survivin as a therapeutic target for radiation sensitization in lung cancer. Cancer Res 64: 2840-2845, 2004

73. Marnett LJ, Kalgutkar AS: Cyclooxygenase 2 inhibitors: discovery, selectivity and the future. Trends Pharmacol Sci 20: 465-469, 1999

74. Masferrer JL: Cyclooxygenase-2 inhibitors in cancer prevention and treatment. Adv Exp Med Biol 532:209-213, 2003

75. Masferrer JL, Leahy KM, Koki AT, et al: Antiangiogenic and antitumor activities of cyclooxygenase- 2 inhibitors. Cancer Res 60:1306-1311, 2000

76. Milas L, Mason KA, Crane $\mathrm{CH}$, et al: Improvement of radiotherapy or chemoradiotherapy by targeting COX-2 enzyme. Oncology (Williston Park) 17 (Suppl 5):15-24, 2003

77. Nakata E, Mason KA, Hunter N, et al: Potentiation of tumor response to radiation or chemoradiation by selective cyclooxygenase-2 enzyme inhibitors. Int J Radiat Oncol Biol Phys 58: 369-375, 2004

78. Nam DH, Park K, Park C, et al: Intracranial inhibition of glioma cell growth by cyclooxygenase- 2 inhibitor celecoxib. Oncol Rep 11:263-268, 2004

79. Niederberger E, Manderscheid C, Grösch S, et al: Effects of the selective COX-2 inhibitors celecoxib and rofecoxib on human vascular cells. Biochem Pharmacol 68:341-350, 2004

80. Nugent FW, Mertens WC, Graziano S, et al: Docetaxel and cyclooxygenase- 2 inhibition with celecoxib for advanced nonsmall cell lung cancer progressing after platinum-based chemotherapy: a multicenter phase II trial. Lung Cancer 48:267-273, 2005

81. Nussmeier NA, Whelton AA, Brown MT, et al: Complications of the COX-2 inhibitors parecoxib and valdecoxib after cardiac surgery. N Engl J Med 352:1081-1091, 2005

82. Olashaw N, Pledger WJ: Paradigms of growth control: relation to Cdk activation. Sci STKE 2002:RE7, 2002

83. Pan CX, Loehrer P, Seitz D, et al: A phase II trial of irinotecan, 5-fluorouracil and leucovorin combined with celecoxib and glutamine as first-line therapy for advanced colorectal cancer. Oncology 69:63-70, 2005

84. Parente L, Perretti M: Advances in the pathophysiology of constitutive and inducible cyclooxygenases: two enzymes in the spotlight. Biochem Pharmacol 65:153-159, 2003

85. Patel MI, Subbaramaiah K, Du B, et al: Celecoxib inhibits prostate cancer growth: evidence of a cyclooxygenase-2-independent mechanism. Clin Cancer Res 11:1999-2007, 2005

86. Paulson SK, Kaprak TA, Gresk CJ, et al: Plasma protein binding of celecoxib in mice, rat, rabbit, dog and human. Biopharm Drug Dispos 20:293-299, 1999

87. Pennati M, Binda M, De Cesare M, et al: Ribozyme-mediated down-regulation of survivin expression sensitizes human melanoma cells to topotecan in vitro and in vivo. Carcinogenesis 25:1129-1136, 2004

88. Penning TD, Talley JJ, Bertenshaw SR, et al: Synthesis and biological evaluation of the 1,5-diarylpyrazole class of cyclooxygenase-2 inhibitors: identification of 4-[5-(4-methylphenyl)-3-(trifluoromethyl)-1 $H$-pyrazol-1-yl]benzenesulfonamide (SC-58635, celecoxib). J Med Chem 40:1347-1365, 1997
89. Petersen C, Petersen S, Milas L, et al: Enhancement of intrinsic tumor cell radiosensitivity induced by a selective cyclooxygenase-2 inhibitor. Clin Cancer Res 6:2513-2520, 2000

90. Pouplana R, Lozano JJ, Pérez C, et al: Structure-based QSAR study on differential inhibition of human prostaglandin endoperoxide $\mathrm{H}$ synthase-2 (COX-2) by nonsteroidal anti-inflammatory drugs. J Comput Aided Mol Des 16:683-709, 2002

91. Prince HM, Mileshkin L, Roberts A, et al: A multicenter phase II trial of thalidomide and celecoxib for patients with relapsed and refractory multiple myeloma. Clin Cancer Res 11:5504-5514, 2005

92. Pyo H, Choy H, Amorino GP, et al: A selective cyclooxygenase-2 inhibitor, NS-398, enhances the effect of radiation in vitro and in vivo preferentially on the cells that express cyclooxygenase-2. Clin Cancer Res 7:2998-3005, 2001

93. Raju U, Nakata E, Yang P, et al: In vitro enhancement of tumor cell radiosensitivity by a selective inhibitor of cyclooxygenase-2 enzyme: mechanistic considerations. Int J Radiat Oncol Biol Phys 54:886-894, 2002

94. Raz A: Is inhibition of cyclooxygenase required for the antitumorigenic effects of nonsteroidal, anti-inflammatory drugs (NSAIDs)? In vitro versus in vivo results and the relevance for the prevention and treatment of cancer. Biochem Pharmacol 63:343-347, 2002

95. Reardon DA, Quinn JA, Vredenburgh J, et al: Phase II trial of irinotecan plus celecoxib in adults with recurrent malignant glioma. Cancer 103:329-338, 2005

96. Rödel F, Hoffmann J, Distel L, et al: Survivin as a radioresistance factor, and prognostic and therapeutic target for radiotherapy in rectal cancer. Cancer Res 65:4881-4887, 2005

97. Roh JL, Sung MW, Park SW, et al: Celecoxib can prevent tumor growth and distant metastasis in postoperative setting. Cancer Res 64:3230-3235, 2004

98. Sawaoka H, Tsuji S, Tsujii M, et al: Cyclooxygenase inhibitors suppress angiogenesis and reduce tumor growth in vivo. Lab Invest 79:1469-1477, 1999

99. Schlegel J, Durchschlag G, Piontek G, et al: Activation of the phosphatidylinositol-3'-kinase/protein kinase B-dependent antiapoptotic pathway plays an important role in the development of radioresistance of human glioma cells. Ann N Y Acad Sci 973:224-227, 2002

100. Shaik MS, Chatterjee A, Jackson T, et al: Enhancement of antitumor activity of docetaxel by celecoxib in lung tumors. Int J Cancer 118:396-404, 2005

101. Shapiro M, Rook AH, Lehrer MS, et al: Novel multimodality biologic response modifier therapy, including bexarotene and long-wave ultraviolet A for a patient with refractory stage IVa cutaneous T-cell lymphoma. J Am Acad Dermatol 47:956961, 2002

102. Shin YK, Park JS, Kim HS, et al: Radiosensitivity enhancement by celecoxib, a cyclooxygenase (COX)-2 selective inhibitor, via COX-2-dependent cell cycle regulation on human cancer cells expressing differential COX-2 levels. Cancer Res 65:9501-9509, 2005

103. Shono T, Tofilon PJ, Bruner JM, et al: Cyclooxygenase-2 expression in human gliomas: prognostic significance and molecular correlations. Cancer Res 61:4375-4381, 2001

104. Smith WL, DeWitt DL, Garavito RM: Cyclooxygenases: structural, cellular, and molecular biology. Annu Rev Biochem 69:145-182, 2000

105. Solomon SD, McMurray JJV, Pfeffer MA, et al: Cardiovascular risk associated with celecoxib in a clinical trial for colorectal adenoma prevention. N Engl J Med 352: 1071-1080, 2005

106. Song X, Lin HP, Johnson AJ, et al: Cyclooxygenase-2, player or spectator in cyclooxygenase-2 inhibitor-induced apoptosis in prostate cancer cells. J Natl Cancer Inst 94:585-591 2002

107. St. Clair WH, Given CA: Stereotactic radiosurgery associated neurotoxicity. Technol Cancer Res Treat 2:147-152, 2003 
108. Stupp R, van den Bent MJ, Hegi ME: Optimal role of temozolomide in the treatment of malignant gliomas. Curr Neurol Neurosci Rep 5:198-206, 2005

109. Subbaramaiah K, Telang N, Ramonetti JT, et al: Transcription of cyclooxygenase-2 is enhanced in transformed mammary epithelial cells. Cancer Res 56:4424-4429, 1996

110. Sun SY, Schroeder CP, Yue P, et al: Enhanced growth inhibition and apoptosis induction in NSCLC cell lines by combination of celecoxib and 4HPR at clinically relevant concentrations. Cancer Biol Ther 4:407-413, 2005

111. Takada Y, Bhardwaj A, Potdar P, et al: Nonsteroidal antiinflammatory agents differ in their ability to suppress NF- $\kappa$ B activation, inhibition of expression of cyclooxygenase- 2 and cyclin D1, and abrogation of tumor cell proliferation. Oncogene 23:9247-9258, 2004

112. Tanaka K, Tomisato W, Hoshino T, et al: Involvement of intracellular $\mathrm{Ca}^{2+}$ levels in nonsteroidal anti-inflammatory drug-induced apoptosis. J Biol Chem 280:31059-31067, 2005

113. Tegeder I, Pfeilschifter J, Geisslinger G: Cyclooxygenaseindependent actions of cyclooxygenase inhibitors. FASEB J 15:2057-2072, 2001

114. Thun MJ, Henley SJ, Patrono C: Nonsteroidal anti-inflammatory drugs as anticancer agents: mechanistic, pharmacologic, and clinical issues. J Natl Cancer Inst 94:252-266, 2002

115. Trifan OC, Durham WF, Salazar VS, et al: Cyclooxygenase2 inhibition with celecoxib enhances antitumor efficacy and reduces diarrhea side effect of CPT-11. Cancer Res 62: 5778-5784, 2002

116. Tsao MN, Mehta MP, Whelan TJ, et al: The American Society for Therapeutic Radiology and Oncology (ASTRO) evidence-based review of the role of radiosurgery for malignant glioma. Int J Radiat Oncol Biol Phys 63:47-55, 2005

117. Tsuji S, Tsujii M, Kawano S, et al: Cyclooxygenase-2 upregulation as a perigenetic change in carcinogenesis. J Exp Clin Cancer Res 20:117-129, 2001

118. Vane JR, Bakhle YS, Botting RM: Cyclooxygenases 1 and 2. Annu Rev Pharmacol Toxicol 38:97-120, 1998

119. Waskewich C, Blumenthal RD, Li H, et al: Celecoxib exhibits the greatest potency amongst cyclooxygenase (COX) inhibitors for growth inhibition of COX-2-negative hematopoietic and epithelial cell lines. Cancer Res 62:2029-2033, 2002

120. Weber A, Casini A, Heine A, et al: Unexpected nanomolar inhibition of carbonic anhydrase by COX-2-selective celecoxib: new pharmacological opportunities due to related binding site recognition. J Med Chem 47:550-557, 2004

121. Weitzner MA, Meyers CA: Cognitive functioning and quality of life in malignant glioma patients: a review of the literature. Psychooncology 6:169-177, 1997

122. Westphal M, Black PM: Perspectives of cellular and molecular neurosurgery. J Neurooncol 70:255-269, 2004

123. Williams CS, Tsujii M, Reese J, et al: Host cyclooxygenase-2 modulates carcinoma growth. J Clin Invest 105:1589-1594, 2000
124. Williams CS, Watson AJM, Sheng H, et al: Celecoxib prevents tumor growth in vivo without toxicity to normal gut: lack of correlation between in vitro and in vivo models. Cancer Res 60:6045-6051, 2000

125. Williams MR, Arthur JSC, Balendran A, et al: The role of 3phosphoinositide-dependent protein kinase 1 in activating AGC kinases defined in embryonic stem cells. Curr Biol 10: 439-448, 2000

126. Wirth LJ, Haddad RI, Lindeman NI, et al: Phase I study of gefitinib plus celecoxib in recurrent or metastatic squamous cell carcinoma of the head and neck. J Clin Oncol 23: 6976-6981, 2005

127. Wu J, Ling X, Pan D, et al: Molecular mechanism of inhibition of survivin transcription by the GC-rich sequence-selective DNA binding antitumor agent, hedamycin: evidence of survivin down-regulation associated with drug sensitivity. J Biol Chem 280:9745-9751, 2005

128. Xie D, Zeng YX, Wang HJ, et al: Expression of cytoplasmic and nuclear survivin in primary and secondary human glioblastoma. Br J Cancer 94:108-114, 2006

129. Yamazaki R, Kusunoki N, Matsuzaki T, et al: Selective cyclooxygenase-2 inhibitors show a differential ability to inhibit proliferation and induce apoptosis of colon adenocarcinoma cells. FEBS Lett 531:278-284, 2002

130. Yonesaka K, Tamura K, Kurata T, et al: Small interfering RNA targeting survivin sensitizes lung cancer cell with mutant p53 to adriamycin. Int J Cancer 118:812-820, 2005

131. Zhang M, Chakravarti A: Novel radiation-enhancing agents in malignant gliomas. Semin Radiat Oncol 16:29-37, 2006

132. Zhang X, Morham SG, Langenbach R, et al: Malignant transformation and antineoplastic actions of nonsteroidal antiinflammatory drugs (NSAIDs) on cyclooxygenase-null embryo fibroblasts. J Exp Med 190:451-460, 1999

133. Zhang Z, Lai GH, Sirica AE: Celecoxib-induced apoptosis in rat cholangiocarcinoma cells mediated by Akt inactivation and Bax translocation. Hepatology 39:1028-1037, 2004

134. Zhu J, Song X, Lin HP, et al: Using cyclooxygenase-2 inhibitors as molecular platforms to develop a new class of apoptosis-inducing agents. J Natl Cancer Inst 94: 1745-1757, 2002

135. Zweifel BS, Davis TW, Ornberg RL, et al: Direct evidence for a role of cyclooxygenase 2-derived prostaglandin $E_{2}$ in human head and neck xenograft tumors. Cancer Res 62: 6706-6711, 2002

Manuscript received January 20, 2006.

Accepted in final form March 16, 2006.

Funding for this work was received from the James H. Zumberge Faculty Research \& Innovation Fund, from Accelerate Brain Cancer Cure $\left(\mathrm{ABC}^{2}\right)$, and from the Margaret E. Early Medical Research Trust.

Address reprint requests to: Axel H. Schönthal, Ph.D., 2011 Zonal Avenue, HMR-405, Los Angeles, California 90089-9094. email: schontha@usc.edu. 